\title{
A GENERALIZATION OF THE ITÔ FORMULA
}

\section{SAID NGOBI}

\author{
Received 12 February 2001
}

\begin{abstract}
The classical Itô formula is generalized to some anticipating processes. The processes we consider are in a Sobolev space which is a subset of the space of square integrable functions over a white noise space. The proof of the result uses white noise techniques.
\end{abstract}

2000 Mathematics Subject Classification: 60Exx, 60Gxx, 60Hxx.

1. Introduction. The well-known Itô formula relies on the fact that the integrands in the Itô processes are nonanticipating. However, in many cases, this essential condition is not satisfied. Take the example of the stochastic integral $\int_{0}^{1} B(1) d B(t), t<1$, where $B(t)$ is a Brownian motion. Clearly, $B(1)$ is anticipating and $\int_{0}^{1} B(1) d B(t)$ cannot be defined as an Itô integral; necessitating an extension.

We are interested in generalizing the Itô formula to anticipating processes in an infinite-dimensional space. For this purpose, we consider the infinite-dimensional space to be the white noise space $\left(\mathscr{Y}^{\prime}(\mathbb{R}), \mu\right)$, where $\mathscr{S}^{\prime}(\mathbb{R})$ is the space of tempered distributions and $\mu$ is the standard Gaussian measure on $\mathscr{S}^{\prime}(\mathbb{R})$. We use the HitsudaSkorokhod integral as the underlying extension of the Itô integral and we prove the formula for processes of the form $\theta(X(t), F)$ where $X(t)$ is a Wiener integral, $\theta \in \mathscr{C}_{b}^{2}\left(\mathbb{R}^{2}\right)$ and $F \in \mathscr{W}^{1 / 2}$, a Sobolev space in the Hilbert space $\left(L^{2}\right) \equiv L^{2}\left(\mathscr{Y}^{\prime}(\mathbb{R}), \mu\right)$.

A number of variations to the formula in this paper exist. Prevault [9] developed a formula which was applied to processes of the form $Y(t)=\theta(X(t), F)$, where $F$ is a smooth random variable depending on the whole trajectory of $(B(t))_{t \in \mathbb{R}^{+}}$, and $(X(t))_{t \in \mathbb{R}^{+}}$is an adapted Itô process. His proof relies on the expression of infinitesimal time changes on Brownian functionals using the Gross Laplacian. In [6], Kuo developed a formula which was applied to processes of the form $Y(t)=\theta(X(t), B(c)), 0 \leq a \leq c \leq$ $b$, with $X(t)$ a Wiener integral. The main tool of his proof is the very important white noise function, the $S$-transform, and the fact that both $X(t)$ and $B(t)$ have Gaussian laws. The proof in this paper is a limiting process of Kuo's proof.

2. Background. In this section, the basic background from white noise analysis are introduced and the interested reader is provided with the relevant references.

2.1. Concept and notations. Let $E$ be a real separable Hilbert space with norm $|\cdot|_{0}$. Let $A$ be a densely defined selfadjoint operator on $E$, whose eigenvalues $\left\{\lambda_{n}\right\}_{n \geq 1}$ satisfy the following conditions:

(i) $1<\lambda_{1} \leq \lambda_{2} \leq \lambda_{3} \leq \cdots$;

(ii) $\sum_{n=1}^{\infty} \lambda_{n}^{-2}<\infty$. 
For any $p \geq 0$, let $\mathscr{E}_{p}$ be the completion of $E$ with respect to the norm $|f|_{p}:=\left|A^{p} f\right|_{0}$. Note that $\mathscr{E}_{p}$ is a Hilbert space with the norm $|\cdot|_{p}$ and $\mathscr{E}_{p} \subset \mathscr{E}_{q}$ for any $p \geq q$. The second condition on the eigenvalues above implies that the inclusion map $i: \mathscr{E}_{p+1} \rightarrow \mathscr{E}_{p}$ is a Hilbert-Schmidt operator.

Let $\mathscr{E}$ be the projective limit of $\left\{\mathscr{E}_{p} ; p \geq 0\right\}$, and let $\mathscr{E}^{\prime}$ be the dual space of $\mathscr{E}$. Then the space $\mathscr{E}=\bigcap_{p \geq 0} \mathscr{E} p$ equipped with the topology given by the family $\left\{|\cdot|_{p}\right\}_{p \geq 0}$ of seminorms is a nuclear space. Hence $\mathscr{E} \subset E \subset \mathscr{E}$ is a Gel'fand triple with the following continuous inclusions:

$$
\mathscr{E} \subset \mathscr{E}_{q} \subset \mathscr{E}_{p} \subset E \subset \mathscr{E}_{p}^{\prime} \subset \mathscr{E}_{q}^{\prime} \subset \mathscr{E}^{\prime}, \quad q \geq p \geq 0
$$

We used the Riesz representation theorem to identify the dual of $E$ with itself.

It can be shown that for all $p \geq 0$, the dual space $\mathscr{E}_{p}^{\prime}$ is isomorphic to $\mathscr{E}_{-}$, which is the completion of the space $E$ with respect to the norm $|f|_{-p}=\left|A^{-p} f\right|_{0}$.

Minlo's theorem allows us to define a unique probability measure $\mu$ on the Borel subsets of $\mathscr{E}^{\prime}$ with the property that for all $f \in \mathscr{E}$, the random variable $\langle\cdot, f\rangle$ is normally distributed with mean 0 and variance $|f|_{0}^{2}$. We are using $\langle\cdot, \cdot\rangle$ to denote the duality between $\mathscr{E}^{\prime}$ and $\mathscr{E}$. This means that the characteristic functional of $\mu$ is given by

$$
\int_{\mathscr{E}^{\prime}} e^{i\langle x, \xi\rangle} d \mu(x)=e^{-(1 / 2)|\xi|_{0}^{2}}, \quad \forall \xi \in \mathscr{E} .
$$

The probability space $\left(\mathscr{E}^{\prime}, \mu\right)$ is called the white noise space. The space $L^{2}\left(\mathscr{E}^{\prime}, \mu\right)$ will be denoted by $\left(L^{2}\right)$; that is, $\left(L^{2}\right)$ is the set of functions $\varphi: \mathscr{E}^{\prime} \rightarrow \mathbb{C}$ such that $\varphi$ is measurable and $\int_{\mathscr{E}^{\prime}}|\varphi(x)|^{2} d \mu(x)<\infty$. If we denote by $E_{\mathcal{C}}$ the complexification of $E$, the Wiener-Itô theorem allows us to associate to each $\varphi \in\left(L^{2}\right)$ a unique sequence $\left\{f_{n}\right\}_{n \geq 0}, f_{n} \in E_{c}^{\hat{\otimes} n}$ and express $\varphi$ as $\varphi=\sum_{n=0}^{\infty} I_{n}\left(f_{n}\right)$ where $I_{n}\left(f_{n}\right)$ is a multiple Wiener integral of order $n$ (see [3]). This decomposition is similar to what is referred to as the Fock-space decomposition as shown in [8].

The $\left(L^{2}\right)$-norm $\|\varphi\|_{0}$ of $\varphi$ is given by

$$
\|\varphi\|_{0}^{2}=\sum_{n=0}^{\infty} n !\left|f_{n}\right|_{0}^{2}
$$

where $|\cdot|_{0}$ denotes the $E_{c}^{\hat{\otimes} n}$-norm induced from the norm $|\cdot|_{0}$ on $E$. For any $p \geq 0$, let $|\cdot|_{p}$ be the $\mathscr{E}_{p, c}^{\otimes} n$-norm induced from the norm $|\cdot|_{p}$ on $\mathscr{E}_{p}$ and define

$$
\|\varphi\|_{p}=\left(\sum_{n=0}^{\infty} n !\left|f_{n}\right|_{p}^{2}\right)^{1 / 2} .
$$

Let

$$
\left(\mathscr{E}_{p}\right)=\left\{\varphi \in\left(L^{2}\right) ;\|\varphi\|_{p}<\infty\right\} .
$$

If $0<p \leq q$, then $\left(\mathscr{E}_{q}\right) \subset\left(\mathscr{E}_{p}\right)$ with the property that for any $q \geq 0$, there exists $p>q$ such that the inclusion map $I_{p, q}:\left(\mathscr{E}_{p}\right) \hookrightarrow\left(\mathscr{E}_{q}\right)$ is a Hilbert-Schmidt operator and $\left\|I_{p, q}\right\|_{\mathrm{HS}}^{2} \leq\left(1-\left\|i_{p, q}\right\|_{\mathrm{HS}}^{2}\right)^{-1}$ where $i_{p, q}$ is the inclusion map from $\mathscr{E}_{p}$ into $\mathscr{E}_{q}$ as noted earlier. 
Analogous to the way $\mathscr{E}$ was defined, we also define $(\mathscr{E})$ as the projective limit of $\{(\mathscr{E} p) ; p \geq 0\}$ and $(\mathscr{E})^{*}$ as the dual space of $(\mathscr{E})$. With the above result, $(\mathscr{E})=\bigcap_{p \geq 0}(\mathscr{E} p)$ with the topology generated by the family $\left\{\|\cdot\|_{p} ; p \geq 0\right\}$ of norms. It is a nuclear space forming the infinite-dimensional Gel'fand triple $(\mathscr{E}) \subset\left(L^{2}\right) \subset(\mathscr{E}) *$. Moreover, we have the following continuous inclusions:

$$
(\mathscr{E}) \subset\left(\mathscr{E}_{q}\right) \subset\left(\mathscr{E}_{p}\right) \subset\left(L^{2}\right) \subset\left(\mathscr{E}_{-p}\right) \subset\left(\mathscr{E}_{-q}\right) \subset(\mathscr{E})^{*}, \quad q \geq p \geq 0 .
$$

We note that by letting $E=L^{2}(\mathbb{R})$, we get $\mathscr{E}^{\prime}=\mathscr{S}^{\prime}(\mathbb{R})$ and obtain the Gel'fand triple $(\mathscr{Y}) \subset\left(L^{2}\right) \subset(\mathscr{Y})^{*}$. The elements in $(\mathscr{E})$ are called test functions on $\mathscr{E}^{\prime}$ while the elements in $(\mathscr{E})^{*}$ are called generalized functions on $\mathscr{E}^{\prime}$. The bilinear pairing between $(\mathscr{E})$ and $(\mathscr{E}) *$ is denoted by $\langle\langle\cdot, \cdot\rangle\rangle$. If $\varphi \in\left(L^{2}\right)$ and $\psi \in(\mathscr{E})$, then $\langle\langle\varphi, \psi\rangle\rangle=(\varphi, \bar{\psi})$, where $(\cdot, \cdot)$ is the inner product on the complex Hilbert space $\left(L^{2}\right)$.

An element $\varphi \in(\mathscr{E})$ has a unique representation as $\varphi=\sum_{n=0}^{\infty} I_{n}\left(f_{n}\right), f_{n} \in \mathscr{E}_{C}^{\hat{\otimes}} n$ with the norm

$$
\|\varphi\|_{p}^{2}=\sum_{n=0}^{\infty} n !\left|f_{n}\right|_{p}^{2}<\infty, \quad \forall p \geq 0 .
$$

Similarly, an element $\phi \in(\mathscr{E}) *$ can be written as $\phi=\sum_{n=0}^{\infty} I_{n}\left(F_{n}\right), F_{n} \in\left(\mathscr{E}_{C}^{\prime}\right)^{\hat{\otimes} n}$ with the norm

$$
\|\phi\|_{-p}^{2}=\sum_{n=0}^{\infty} n !\left|F_{n}\right|_{-p}^{2}, \quad \text { for some } p \geq 0 .
$$

The bilinear pairing between $\phi$ and $\varphi$ is then represented as

$$
\langle\langle\phi, \varphi\rangle\rangle=\sum_{n=0}^{\infty} n !\left\langle F_{n}, f_{n}\right\rangle .
$$

It is possible to construct wider Gel'fand triples than the one above; for example, by Kondratiev and Streit (see [6, Chapter 4] and the Cochran-Kuo-Sengupta (CKS) space in [1]).

2.2. Hermite polynomials, Wick tensors, and multiple Wiener integrals. The Hermite polynomial of degree $n$ with parameter $\sigma^{2}$ is defined by

$$
: x^{n}:_{\sigma^{2}}=\left(-\sigma^{2}\right)^{n} e^{x^{2} / 2 \sigma^{2}} D_{x}^{n} e^{-x^{2} / 2 \sigma^{2}} .
$$

These polynomials have a generating function given by

$$
\sum_{n=0}^{\infty} \frac{t^{n}}{n !}: x^{n}: \sigma_{\sigma^{2}}=e^{t x-(1 / 2) \sigma^{2} t^{2}} .
$$

The following formulas are also helpful:

$$
\begin{aligned}
: x^{n}:_{\sigma^{2}} & =\sum_{k=0}^{[n / 2]}\left(\begin{array}{c}
n \\
2 k
\end{array}\right)(2 k-1) ! !\left(-\sigma^{2}\right)^{k} x^{n-2 k}, \\
x^{n} & =\sum_{k=0}^{[n / 2]}\left(\begin{array}{c}
n \\
2 k
\end{array}\right)(2 k-1) ! ! \sigma^{2 k}: x^{n-2 k}: \sigma^{2},
\end{aligned}
$$

where $(2 k-1) ! !=(2 k-1)(2 k-3) \cdots 3 \cdot 1$ with $(-1) ! !=1$. 
The trace operator is the element $\tau \in\left(\mathscr{E}_{C}^{\prime}\right)^{\hat{\otimes} 2}$ defined by

$$
\langle\tau, \xi \otimes \eta\rangle=\langle\xi, \eta\rangle, \quad \xi, \eta \in \mathscr{E}_{C} .
$$

Let $x \in \mathscr{E}^{\prime}$. The Wick tensor : $x^{\otimes n}$ : of an element $x$ is defined as

$$
: x^{\otimes n}:=\sum_{k=0}^{[n / 2]}\left(\begin{array}{c}
n \\
2 k
\end{array}\right)(2 k-1) ! !(-1)^{k} x^{\otimes(n-2 k)} \hat{\otimes} T^{\otimes k},
$$

where $\tau$ is the trace operator. The following formula similar to (2.12) is also important for Wick tensors, that is,

$$
x^{\otimes n}=\sum_{k=0}^{[n / 2]}\left(\begin{array}{c}
n \\
2 k
\end{array}\right)(2 k-1) ! !: x^{\otimes(n-2 k)}: \hat{\otimes} \tau^{k} .
$$

For $x \in \mathscr{E}^{\prime}$ and $\xi \in \mathscr{E}$, the following equalities related to Wick tensors hold:

$$
\left\langle: x^{\otimes n}:, \xi^{\otimes n}\right\rangle=:\langle x, \xi\rangle^{n}:|\xi|_{0}^{2}, \quad\left\|\left\langle: x^{\otimes n}:, \xi^{\otimes n}\right\rangle\right\|_{0}=\sqrt{n !}|\xi|_{0}^{n} .
$$

In order to make mathematical computations concerning multiple Wiener integrals easier, they are expressed in terms of Wick tensors. This is achieved via two statements as follows (see [6, Theorem 5.4]):

(1) let $h_{1}, h_{2}, \ldots \in E$ be orthogonal and let $n_{1}+n_{2}+\cdots=n$. Then for almost all $x \in \mathscr{E}^{\prime}$, we have

$$
\left\langle: x^{\otimes n}:, h_{1}^{\otimes n_{1}} \hat{\otimes} h_{2}^{\otimes n_{2}} \hat{\otimes} \cdots\right\rangle=:\left\langle x, h_{1}\right\rangle^{n_{1}}:_{\left|h_{1}\right|_{0}^{2}}:\left\langle x, h_{2}\right\rangle^{n_{2}}:_{\left|h_{2}\right|_{0}^{2}} \cdots ;
$$

(2) let $f \in E_{\mathcal{C}}^{\hat{\otimes} n}$. Then for almost all $x \in \mathscr{E}^{\prime}$,

$$
I_{n}(f)(x)=\left\langle: x^{\otimes n}:, f\right\rangle
$$

where $I_{n}(f)(x)$ is a multiple Wiener integral of order $n$. With this relationship, we are able to write test functions and generalized functions in terms of Wick tensors as follows: any element $\varphi \in\left(L^{2}\right)$ can be expressed as

$$
\varphi(x)=\sum_{n=0}^{\infty}\left\langle: x^{\otimes n}:, f_{n}\right\rangle, \quad \mu \text {-a.e. for } x \in \mathscr{E}^{\prime}, f_{n} \in E_{c}^{\hat{\otimes} n} .
$$

Suppose $\varphi \in(\mathscr{E})$. Since $\mathscr{E}_{C}^{\hat{\otimes}} n$ is dense in $E_{\mathcal{C}}^{\hat{\otimes} n}$, we have

$$
\varphi(x)=\sum_{n=0}^{\infty}\left\langle: x^{\otimes n}:, f_{n}\right\rangle, \quad f_{n} \in \mathscr{E}_{c}^{\hat{\otimes} n} .
$$

For an element $\phi \in\left(\mathscr{E}_{p}\right)^{*}$, it follows that

$$
\phi(x)=\sum_{n=0}^{\infty}\left\langle: x^{\otimes n}:, F_{n}\right\rangle, \quad F_{n} \in\left(\mathscr{E}_{p}^{\prime}\right)^{\hat{\otimes} n} .
$$


2.3. The white noise differential operator. Let $y \in \mathscr{E}^{\prime}$ and $\varphi=\left\langle: x^{\otimes n}:, f\right\rangle \in(\mathscr{E})$. It can be shown that the directional derivative

$$
\lim _{t \rightarrow 0} \frac{\varphi(x+t y)-\varphi(x)}{t}=n\left\langle: x^{\otimes(n-1)}:, y \hat{\otimes}_{1} f\right\rangle,
$$

where $y \hat{\otimes}_{1}$ is the unique continuous and linear map from $E_{c}^{\hat{\otimes} n}$ into $E_{c}^{\hat{\otimes}(n-1)}$ such that

$$
y \hat{\otimes}_{1} g^{\otimes n}=\langle y, g\rangle g^{\otimes(n-1)}, \quad g \in E_{c} .
$$

This shows that the function $\varphi$ has Gâteaux derivative $D_{y} \varphi$. For general $\varphi(x)=$ $\sum_{n=0}^{\infty}\left\langle: x^{\otimes n}:, f_{n}\right\rangle, f_{n} \in \mathscr{E}_{C}^{\otimes} \hat{n}$, we define an operator $D_{y}$ on $(\mathscr{E})$ as

$$
D_{y} \varphi(x) \equiv \sum_{n=1}^{\infty} n\left\langle: x^{\otimes(n-1)}:, y \hat{\otimes}_{1} f_{n}\right\rangle .
$$

It can be shown that $D_{y}$ is a continuous linear operator on ( $\left.\mathscr{E}\right)$ (see [6, Section 9.1]). By the duality between $(\mathscr{E}) *$ and $(\mathscr{E})$, the adjoint operator $D_{y}^{*}$ of $D_{y}$ can be defined by

$$
\left\langle\left\langle D_{y}^{*} \Phi, \varphi\right\rangle\right\rangle=\left\langle\left\langle\Phi, D_{y} \varphi\right\rangle\right\rangle, \quad \Phi \in(\mathscr{E})^{*}, \varphi \in(\mathscr{E}) .
$$

Now let $\mathscr{E}$ be the Schwartz space of all infinitely differentiable functions $f: \mathbb{R} \rightarrow \mathbb{R}$ such that for all $n, k \in \mathbb{N}$,

$$
\sup _{x \in \mathbb{R}}\left|x^{n}\left(\frac{d^{k}}{d x^{k}}\right) f(x)\right|<\infty .
$$

If we take $y=\delta_{t}$, the Dirac delta function at $t$, then

(1) $\partial_{t} \equiv D_{\delta_{t}}$ is called the white noise differential operator, the Hida differential operator, or the annihilation operator,

(2) $\partial_{t}^{*} \equiv D_{\delta_{t}}^{*}$ is called the creation operator,

(3) for $\varphi \in(\mathscr{\varphi}), \dot{B}(t) \varphi \equiv \partial_{t} \varphi+\partial_{t}^{*} \varphi$ is called white noise multiplication.

2.4. The $S$-transform. Let $\Phi \in(\mathscr{E}) *$. The $S$-transform is a function on $\mathscr{E}_{c}$ defined by

$$
S(\Phi)(\xi)=\left\langle\left\langle\Phi,: e^{\langle\cdot, \xi\rangle}:\right\rangle\right\rangle, \quad \xi \in \mathscr{E}_{C} .
$$

The $S$-transform is an injective function because the exponential functions span a dense subset of ( $\mathscr{Y})$. If $\Phi \in\left(L^{2}\right)$, the $S$-transform of $\Phi$ is also called the Segal-Bargmann transform of $\Phi$.

3. White noise integrals. From now on, our reference Gel'fand triple is $(\mathscr{E})_{\beta} \subset$ $\left(L^{2}\right) \subset(\mathscr{E})_{\beta}^{*}$. A white noise integral is a type of integral for which the integrand takes values in the space $(\mathscr{E})_{\beta}^{*}$ of generalized functions. As an example, consider the integral $\int_{0}^{t} e^{-c(t-s)}: \dot{B}(s)^{2}: d s$ where $\dot{B}$ is white noise. In this case, the integrand is an $(\mathscr{Y})_{\beta}^{*}$ valued measurable function on $[0, t]$. A second example is the integral $\int_{0}^{t} \partial_{s}^{*} \Phi(s) d s$ where $\Phi$ is also $(\mathscr{T})_{\beta}^{*}$-valued. If $\int_{0}^{t} \partial_{s}^{*} \Phi(s) d s$ is a random variable in $\left(L^{2}\right)$, then the white noise integral is called a Hitsuda-Skorokhod integral. So in general, if $\Phi$ is an 
$(\mathscr{E})_{\beta}^{*}$-valued function on a measurable space $(M, \mathscr{B}, m)$, a white noise integral is an integral of the type

$$
\int_{E} \Phi(u) d m(u), \quad E \in \mathscr{B} .
$$

Despite the fact that $(\mathscr{E})_{\beta}^{*}$ is not a Banach space, these integrals can be defined in the Pettis or Bochner sense by the use of the $S$-transform.

3.1. White noise integrals in the Pettis sense. We need to define $\int_{E} \Phi(u) d m(u)$ as the generalized function in $(\mathscr{E})_{\beta}^{*}$ that satisfies the following:

$$
S\left(\int_{E} \Phi(u) d m(u)\right)(\xi)=\int_{E} S(\Phi(u))(\xi) d m(u), \quad \xi \in \mathscr{E}_{c} .
$$

In particular, if $\Phi(u)$ is replaced by $\partial_{u}^{*} \Phi(u)$, we have

$$
S\left(\int_{E} \partial_{u}^{*} \Phi(u) d m(u)\right)(\xi)=\int_{E} \xi(u) S(\Phi(u))(\xi) d m(u), \quad \xi \in \mathscr{E}_{C} .
$$

The above two equations then call for the following conditions on the function $\Phi$ to be satisfied:

(a) $S(\Phi(u))(\xi)$ is measurable for any $\xi \in \mathscr{E}_{c}$;

(b) $S(\Phi(\cdot))(\xi) \in L^{1}(M)$ for any $\xi \in \mathscr{E}_{C}$;

(c) for any $E \in \mathscr{B}$, the function $\int_{E} S(\Phi(u))(\cdot) d m(u)$ is a generalized function in $(\mathscr{E})_{\beta}^{*}$. (This can be verified by using the characterization theorem for generalized functions.)

The statement in (c) can be rewritten as

$$
\left\langle\left\langle\int_{E} \Phi(u) d m(u),: e^{\langle\cdot, \xi\rangle}:\right\rangle\right\rangle=\int_{E}\left\langle\left\langle\Phi(u),: e^{\langle\cdot, \xi\rangle}:\right\rangle\right\rangle d m(u), \quad \xi \in \mathscr{E}_{c} .
$$

Since the linear span of the set $\left\{: e^{\langle\cdot, \xi\rangle}:, \xi \in \mathscr{E}_{C}\right\}$ is dense in $(\mathscr{E})_{\beta}^{*}$, the above equation implies that

$$
\left\langle\left\langle\int_{E} \Phi(u) d m(u), \varphi\right\rangle\right\rangle=\int_{E}\langle\langle\Phi(u), \varphi\rangle\rangle d m(u), \quad \varphi \in(\mathscr{E})_{\beta}^{*}
$$

In terms of the $S$-transform, Pettis integrability can be characterized using the following theorem. For a proof, see [6, Section 13.4].

THEOrem 3.1. Suppose that the function $\Phi: M \rightarrow(\mathscr{E}){ }_{\beta}^{*}$ satisfies the following conditions:

(1) $S(\Phi(\cdot))(\xi)$ is measurable for any $\xi \in \mathscr{E}_{c}$;

(2) there exists nonnegative numbers $K, a$, and $p$ such that

$$
\int_{M} S(\Phi(u))(\xi) d m(u) \leq K \exp \left[a|\xi|_{p}^{2 /(1-\beta)}\right], \quad \xi \in \mathscr{E}_{c} .
$$


Then $\Phi$ is Pettis integrable and for any $E \in \mathscr{B}$,

$$
S\left(\int_{E} \Phi(u) d m(u)\right)(\xi)=\int_{E} S(\Phi(u))(\xi) d m(u), \quad \xi \in \mathscr{E}_{C} .
$$

3.2. White noise integrals in the Bochner sense. We know that $(\mathscr{E})_{\beta}^{*}$ is not a Banach space but $(\mathscr{E})_{\beta}^{*}=\cup_{p \geq 0}\left(\mathscr{E}_{p}\right)_{\beta}^{*}$ and each of the spaces $\left(\mathscr{E}_{p}\right)_{\beta}^{*}$ is a separable Hilbert space. With this in mind, the white noise integral $\int_{E} \Phi(u) d m(u)$ can be defined in the Bochner sense in the following way. Let $\Phi: M \rightarrow(\mathscr{E})_{\beta}^{*}$, then $\Phi$ is Bochner integrable if it satisfies the following conditions:

(1) $\Phi$ is weakly measurable;

(2) there exists $p \geq 0$ such that $\Phi(u) \in\left(\mathscr{E}_{p}\right)_{\beta}^{*}$ for almost all $u \in M$ and $\|\Phi(\cdot)\|_{-p,-\beta} \in$ $L^{1}(M)$.

If $\Phi$ is Bochner integrable, then we have

$$
\left\|\int_{M} \Phi(u) d m(u)\right\|_{-p,-\beta} \leq \int_{M}\|\Phi(u)\|_{-p,-\beta} d m(u) .
$$

The following theorem contains the conditions for Bochner integrability in terms of the $S$-transform and helps estimate the norm $\|\Phi(u)\|_{-p,-\beta}$ of $\Phi$. See [6, Section 13.5].

THEOREM 3.2. Let $\Phi: M \rightarrow(\mathscr{E})_{\beta}^{*}$ be a function satisfying the following conditions:

(1) $S(\Phi(\cdot))(\xi)$ is measurable for any $\xi \in \mathscr{E}_{c}$;

(2) there exists $p \geq 0$ and nonnegative functions $L \in L^{1}(M), b \in L^{\infty}(M)$, and an $m$-null set $E_{0}$ such that

$$
|S(\Phi(u))(\xi)| \leq L(u) \exp \left[b(u)|\xi|_{p}^{2 /(1-\beta)}\right], \quad \forall \xi \in \mathscr{E}_{c}, u \in E_{0}^{c} .
$$

Then $\Phi$ is Bochner integrable and $\int_{M} \Phi(u) d m(u) \in\left(\mathscr{E}_{q}\right)_{\beta}^{*}$ for any $q>p$ such that

$$
e^{2}\left(\frac{2\|b\|_{\infty}}{1-\beta}\right)^{1-\beta}\left\|A^{-(q-p)}\right\|_{\mathrm{HS}}^{2}<1,
$$

where $\|b\|_{\infty}$ is the essential supremum of $b$. It turns out that for such $q$,

$$
\left\|\int_{M} \Phi(u) d m(u)\right\|_{-q,-\beta} \leq\|L\|_{1}\left(1-e^{2}\left(\frac{2\|b\|_{\infty}}{1-\beta}\right)\left\|A^{-(q-p)}\right\|_{\mathrm{HS}}^{2}\right)^{-1 / 2} .
$$

An example worth noting is the following. Let $F \in \mathscr{S}^{\prime}(\mathbb{R})$ and $f \in L^{2}(\mathbb{R}), f \neq 0$. Then $F(\langle\cdot, f\rangle)$ is a generalized function and if the Fourier transform $\hat{F} \in L^{\infty}(\mathbb{R})$, then $F(\langle\cdot, f\rangle)$ is represented as a white noise integral by

$$
F(\langle\cdot, f\rangle)=\frac{1}{\sqrt{2 \pi}} \int_{\mathbb{R}} e^{i u\langle\cdot, f\rangle} \hat{F}(u) d u .
$$

In this case, $\Phi(u)=e^{i u\langle\cdot, f\rangle} \hat{F}(u), u \in \mathbb{R}$ and it satisfies the conditions in Theorem 3.1 for Pettis integrability. 
4. An extension of the Itô integral. A number of extensions of the Itô integral exist. One such extension is by Itô [4], where he extended it to stochastic integrals for integrands which may be anticipating. In particular, he showed that $\int_{0}^{1} B(1) d B(t)=$ $B(1)^{2}$. In [2], a special type of integral called the Hitsuda-Skorokhod integral (see Definition 4.1 below) was introduced as a motivation to obtain an Itô type formula for such functions as $\theta(B(t), B(c)), t<c$, for a $\mathscr{b}^{2}$-function $\theta$. (We note here that $B(c)$, $t<c$, is not $\mathscr{F}_{t}$ measurable.)

Consider the Gel'fand triple $(\mathscr{Y})_{\beta} \subset\left(L^{2}\right) \subset(\mathscr{Y})_{\beta}^{*}$ which comes from the Gel'fand triple $\mathscr{Y}(\mathbb{R}) \subset L^{2}(\mathbb{R}) \subset \mathscr{Y}^{\prime}(\mathbb{R})$ as described in Section 2.1. Suppose $\Phi:[a, b] \rightarrow(\mathscr{Y})_{\beta}^{*}$ is Pettis integrable. Then the function $t \rightarrow \partial_{t}^{*} \Phi(t)$ is also Pettis integrable and (3.3) holds.

Definition 4.1. Let $\varphi:[a, b] \rightarrow(\mathscr{Y})_{\beta}^{*}$ be Pettis integrable. The white noise integral $\int_{a}^{b} \partial_{t}^{*} \varphi(t) d t$ is called the Hitsuda-Skorokhod integral of $\varphi$ if it is a random variable in $\left(L^{2}\right)$.

Consider a stochastic process $\varphi(t)$ in the space $L^{2}\left([a, b] \times \mathscr{Y}^{\prime}(\mathbb{R})\right)$ which is nonanticipating. The Itô integral $\int_{a}^{b} \varphi(t) d B(t)$ for the process $\varphi(t)$ can be expressed as a white noise integral in the Pettis sense. The following theorem due to Kubo and Takenaka [5] (see also [6, Theorem 13.12] for a proof) implies that the Hitsuda-Skorokhod integral is an extension of the Itô integral to $\varphi(t)$ which might be anticipating. A look at Example 4.3 will throw some light on the difference between Itô's extension and the Hitsuda-Skorokhod integral.

THEOREM 4.2. Let $\varphi(t)$ be nonanticipating and $\int_{a}^{b}\|\varphi(t)\|_{0}^{2} d t<\infty$. Then the function $\partial_{t}^{*} \varphi(t), t \in[a, b]$, is Pettis integrable and

$$
\int_{a}^{b} \partial_{t}^{*} \varphi(t) d t=\int_{a}^{b} \varphi(t) d B(t),
$$

where the right-hand side is the Itô integral of $\varphi$.

EXAMPLE 4.3. Let $\varphi(t)=B(1), t \leq 1$. It was observed earlier that by Itô's extension, $\int_{0}^{1} B(1) d B(t)=B(1)^{2}$. However, for the Hitsuda-Skorokhod integral, $\int_{0}^{1} \partial_{t}^{*} B(1) d t=$ $B(1)^{2}-1$. This equality can be verified by using the $S$-transform in the following way.

Since $B(1)=\left\langle\cdot, 1_{[0,1)}\right\rangle$, we have $(S B(1))(\xi)=\int_{0}^{1} \xi(s) d s$. Now, by the use of (3.3),

$$
\begin{aligned}
S\left(\int_{0}^{1} \partial_{t}^{*} B(1) d t\right)(\xi) & =\int_{0}^{1} \xi(t)(S B(1))(\xi) d t \\
& =\int_{0}^{1} \int_{0}^{1} \xi(t) \xi(s) d t d s \\
& =S\left\langle: \cdot^{\otimes 2}:, 1_{[0,1)}^{\otimes 2}\right\rangle(\xi) .
\end{aligned}
$$

Therefore, $\int_{0}^{1} \partial_{t}^{*} B(1) d t=\left\langle: \otimes^{\otimes 2}:, 1_{[0,1)}^{\otimes 2}\right\rangle$. We can apply the results from Section 2.2 concerning Wick tensors to get

$$
\begin{aligned}
\int_{0}^{1} \partial_{t}^{*} B(1) d t & =:\left\langle\cdot, 1_{[0,1)}\right\rangle^{2}:_{1} \\
& =\left\langle\cdot, 1_{[0,1)}\right\rangle^{2}-1 \\
& =B(1)^{2}-1
\end{aligned}
$$


We clearly see a difference between the integral $\int_{0}^{1} B(1) d B(t)$ as defined by Itô and the Hitsuda-Skorokhod integral of $B(1)$.

DeFINITION 4.4. For $\varphi=\sum_{n=0}^{\infty}\left\langle:{ }^{\otimes n}:, f_{n}\right\rangle$, we define

$$
N \varphi=\sum_{n=1}^{\infty} n\left\langle:{ }^{\otimes n}:, f_{n}\right\rangle .
$$

The operator $N$ is called the number operator. Moreover, the power $N^{r}, r \in \mathbb{R}$, of the number operator is defined in the following way: for $\varphi=\sum_{n=0}^{\infty}\left\langle: \cdot{ }^{\otimes n}:, f_{n}\right\rangle$,

$$
N^{r} \varphi=\sum_{n=1}^{\infty} n^{r}\left\langle: \cdot{ }^{\otimes n}:, f_{n}\right\rangle .
$$

For any $r \in \mathbb{R}, N^{r}$ is a continuous linear operator from $(\mathscr{Y})_{\beta}$ into itself and from $(\mathscr{S})_{\beta}^{*}$ into itself. Let $\mathscr{W}^{1 / 2}$ be the Sobolev space of order $1 / 2$ for the Gel'fand triple $(\mathscr{S})_{\beta} \subset\left(L^{2}\right) \subset(\mathscr{S})_{\beta}^{*}$. In other words, for $[a, b] \subset \mathbb{R}^{+}, \mathscr{W}^{1 / 2}$ will denote the set of $\varphi \in\left(L^{2}\right)$ such that $\left(\partial_{t} \varphi\right)_{t \in[a, b]} \in L^{2}\left([a, b] ;\left(L^{2}\right)\right)$. The norm on $\mathcal{W}^{1 / 2}$ will be defined as

$$
\begin{aligned}
\|\varphi\|_{1 / 2}^{2} & =\left\|(N+1)^{1 / 2} \varphi\right\|_{0}^{2} \\
& =\left\|\sum_{n=0}^{\infty}(n+1)^{1 / 2}\left\langle: \cdot{ }^{\otimes n}:, f_{n}\right\rangle\right\|_{0}^{2} \\
& =\sum_{n=0}^{\infty} n !(n+1)\left|f_{n}\right|_{0}^{2} \\
& =\sum_{n=0}^{\infty} n !\left|f_{n}\right|_{0}^{2}+\sum_{n=0}^{\infty} n ! n\left|f_{n}\right|_{0}^{2} \\
& =\|\varphi\|_{0}^{2}+\left\|N^{1 / 2} \varphi\right\|_{0}^{2} .
\end{aligned}
$$

Now if $\varphi$ is given with the property that $N^{1 / 2} \varphi \in\left(L^{2}\right)$, and $t \in[a, b]$, then $\left\|N^{1 / 2} \varphi\right\|_{0}^{2}=$ $\int_{a}^{b}\left\|\partial_{t} \varphi\right\|_{0}^{2} d t$. Hence,

$$
\|\varphi\|_{1 / 2}^{2}=\|\varphi\|_{0}^{2}+\int_{a}^{b}\left\|\partial_{t} \varphi\right\|_{0}^{2} d t
$$

and so

$$
W^{1 / 2} \equiv\left\{\varphi \in\left(L^{2}\right) ;\|\varphi\|_{1 / 2}<\infty\right\} .
$$

For more information on Sobolev spaces and the Number operator, see [7].

The following theorem gives a condition on the function $\varphi(t)$ in order for $\int_{a}^{b} \partial_{t}^{*} \varphi(t) d t$ to be a Hitsuda-Skorokhod integral. This condition is determined by the number operator $N$ and the space $\mathcal{W}^{1 / 2}$ plays a major role. The proof for this condition can be found in [6, Theorem 13.16]. We first identify the space $L^{2}\left([a, b] ;\left(L^{2}\right)\right)$ with the space $L^{2}\left([a, b] \times \mathscr{Y}^{\prime}\right)$. 
THEOREM 4.5. Let $\varphi \in L^{2}\left([a, b] ; W^{1 / 2}\right)$, then $\int_{a}^{b} \partial_{t}^{*} \varphi(t) d t$ is a Hitsuda-Skorokhod integral and

$$
\left\|\int_{a}^{b} \partial_{t}^{*} \varphi(t) d t\right\|_{0}^{2}=\int_{a}^{b}\|\varphi(t)\|_{0}^{2} d t+\int_{a}^{b} \int_{a}^{b}\left(\left(\partial_{t} \varphi(s), \partial_{s} \varphi(t)\right)\right)_{0} d s d t
$$

where $((\cdot, \cdot))_{0}$ is the inner product on $\left(L^{2}\right)$. Moreover,

$$
\left|\int_{a}^{b} \int_{a}^{b}\left(\left(\partial_{t} \varphi(s), \partial_{s} \varphi(t)\right)\right)_{0} d s d t\right| \leq \int_{a}^{b}\left\|N^{1 / 2} \varphi(t)\right\|_{0}^{2} d t .
$$

As a remark, when $\varphi \in L^{2}\left([a, b] ;\left(L^{2}\right)\right)$ is nonanticipating, then the inner product $\left(\left(\partial_{t} \varphi(s), \partial_{s} \varphi(t)\right)\right)_{0}=0$ for almost all $(s, t) \in[a, b]^{2}$. Therefore, when attempting to compute the $L^{2}$-norm for the integral by using (4.9), we obtain the following very useful result that relates the norms:

$$
\left\|\int_{a}^{b} \partial_{t}^{*} \varphi(t) d t\right\|_{0}^{2}=\int_{a}^{b}\|\varphi(t)\|_{0}^{2} d t=\left\|\int_{a}^{b} \varphi(t) d B(t)\right\|_{0}^{2} .
$$

The Hitsuda-Skorokhod integral is related to two other extensions of the Itô integral: the forward and backward integrals. For $\varphi \in L^{2}\left([a, b] ; W^{1 / 2}\right)$, let $\partial_{t^{+}} \varphi(t)$ and $\partial_{t^{-}} \varphi(t)$ be the right-hand white noise derivative and left-hand white noise derivative of $\varphi$, respectively (see [6, Definition 13.25]). The forward integral of $\varphi$ is defined as

$$
\int_{a}^{b} \varphi(t) d B\left(t^{+}\right) \equiv \int_{a}^{b} \partial_{t^{+}} \varphi(t) d t+\int_{a}^{b} \partial_{t}^{*} \varphi(t) d t,
$$

provided that both integrals on the right-hand side are random variables in $\left(L^{2}\right)$. In particular,

$$
\int_{0}^{1} B(1) d B\left(t^{+}\right)=B(1)^{2}
$$

which agrees with $\int_{0}^{1} B(1) d B(t)=B(1)^{2}$ as shown by Itô's in [4]. Similarly, the backward integral of $\varphi$ is defined as

$$
\int_{a}^{b} \varphi(t) d B\left(t^{-}\right) \equiv \int_{a}^{b} \partial_{t^{-}} \varphi(t) d t+\int_{a}^{b} \partial_{t}^{*} \varphi(t) d t
$$

5. An anticipating Itô formula. In this section, the main result is presented. It is about a particular generalization of the ordinary Itô formula to a class of functions that are anticipating.

Let $B(t)$ be a Brownian motion given by $B(t)=\left\langle\cdot, 1_{[0, t)}\right\rangle$. For a $\mathscr{C}^{2}$-function $\theta$, a simple case of the Itô formula is given by

$$
\theta(B(t))=\theta(B(a))+\int_{a}^{t} \theta^{\prime}(B(s)) d B(s)+\frac{1}{2} \int_{a}^{t} \theta^{\prime \prime}(B(s)) d s,
$$

where $0 \leq a \leq t$. 
If we assume that $\theta(B(\cdot)), \theta^{\prime}(B(\cdot)), \theta^{\prime \prime}(B(\cdot)) \in L^{2}\left([a, b] ;\left(L^{2}\right)\right)$, then Theorem 4.2 enables us to write the above equality as

$$
\theta(B(t))=\theta(B(a))+\int_{a}^{t} \partial_{s}^{*} \theta^{\prime}(B(s)) d s+\frac{1}{2} \int_{a}^{t} \theta^{\prime \prime}(B(s)) d s,
$$

where $\int_{0}^{t} \partial_{s}^{*} \theta^{\prime}(B(s)) d s$ is a Hitsuda-Skorokhod integral. In [6], the following two generalizations of the white noise version of the Itô formula in (5.2) were considered:

(a) $\theta(X(t), B(c))$ for a $\mathscr{C}^{2}$-function $\theta$ and a Wiener integral $X(t), t \leq c$;

(b) $\theta(B(t))$ with a generalized function $\theta$ in $\mathscr{Y}^{\prime}(\mathbb{R})$.

The main tool for the proofs of the formulas obtained for the above two generalizations is the $S$-transform. The result for the generalization of (a) is stated below as a theorem and as earlier explained, my new formula will be using this particular generalization. (See [6, Theorem 13.21] for a complete proof.)

THEOREM 5.1. Let $0 \leq a \leq c \leq b$, let $X(t)=\int_{a}^{t} f(s) d B(s)$ be a Wiener integral with $f \in L^{\infty}([a, b])$ and let $\theta(x, y)$ be $a \mathscr{C}^{2}$-function on $\mathbb{R}^{2}$ such that

$$
\theta(X(\cdot), B(c)), \quad \frac{\partial^{2} \theta}{\partial x^{2}}(X(\cdot), B(c)), \quad \frac{\partial^{2} \theta}{\partial x \partial y}(X(\cdot), B(c))
$$

are all in $L^{2}\left([a, b] ;\left(L^{2}\right)\right)$. Then for any $a \leq t \leq b$, the integral

$$
\int_{a}^{t} \partial_{s}^{*}\left(f(s) \frac{\partial \theta}{\partial x}(X(s), B(c))\right) d s
$$

is a Hitsuda-Skorokhod integral and the following equalities hold in $\left(L^{2}\right)$ :

(1) for $a \leq t \leq c$,

$$
\begin{aligned}
\theta(X(t), B(c))= & \theta(X(a), B(c))+\int_{a}^{t} \partial_{s}^{*}\left(f(s) \frac{\partial \theta}{\partial x}(X(s), B(c))\right) d s \\
& +\int_{a}^{t}\left(\frac{1}{2} f(s)^{2} \frac{\partial^{2} \theta}{\partial x^{2}}(X(s), B(c))+f(s) \frac{\partial^{2} \theta}{\partial x \partial y}(X(s), B(c))\right) d s,
\end{aligned}
$$

(2) for $c<t \leq b$,

$$
\begin{aligned}
\theta(X(t), B(c))= & \theta(X(a), B(c))+\int_{a}^{t} \partial_{s}^{*}\left(f(s) \frac{\partial \theta}{\partial x}(X(s), B(c))\right) d s \\
& +\frac{1}{2} \int_{a}^{t} f(s)^{2} \frac{\partial^{2} \theta}{\partial x^{2}}(X(s), B(c)) d s+\int_{a}^{c} f(s) \frac{\partial^{2} \theta}{\partial x \partial y}(X(s), B(c)) d s .
\end{aligned}
$$

As hinted earlier, the proof of the main result in this paper is a limiting process of the above theorem. The following is the main result.

THEOREM 5.2. Let $f \in L^{\infty}([a, b])$ and $X(t)=\int_{a}^{t} f(s) d B(s)$ be a Wiener integral. Let $F \in \mathscr{W}^{1 / 2}$ and $\theta \in \mathscr{C}_{b}^{2}\left(\mathbb{R}^{2}\right)$ such that

$$
\theta(X((\cdot), F)), \frac{\partial^{2} \theta}{\partial x^{2}}(X(\cdot), F), \frac{\partial^{2} \theta}{\partial x \partial y}(X(\cdot), F)
$$


are all in $L^{2}\left([a, b] ;\left(L^{2}\right)\right)$. Then for any $a \leq t \leq b$, the integral

$$
\int_{a}^{t} \partial_{s}^{*}\left(f(s) \frac{\partial \theta}{\partial x}(X(s), F)\right) d s
$$

is a Hitsuda-Skorokhod integral and the following equality holds in $\left(L^{2}\right)$ :

$$
\begin{aligned}
\theta(X(t), F)= & \theta(X(a), F)+\int_{a}^{t} \partial_{s}^{*}\left(f(s) \frac{\partial \theta}{\partial x}(X(s), F)\right) d s \\
& +\frac{1}{2} \int_{a}^{t} f(s)^{2} \frac{\partial^{2} \theta}{\partial x^{2}}(X(s), F) d s+\int_{a}^{t} f(s)\left(\partial_{s} F\right) \frac{\partial^{2} \theta}{\partial x \partial y}(X(s), F) d s
\end{aligned}
$$

The proof is presented in the following steps. First it will be shown that for $F=$ $B(c), 0 \leq a \leq c \leq b$ the above formula holds and that it coincides with the formula in Theorem 3.1. Secondly a special choice of $F$ will be taken in the following way: we know that the span of the set $\left\{: e^{\langle\cdot, g\rangle}: ; g \in L^{2}(\mathbb{R})\right\}$ is dense in $\left(L^{2}\right)$. If we let $g_{1}, g_{2}, \ldots, g_{k} \in L^{2}(\mathbb{R})$ and

$$
\begin{aligned}
F_{N}= & \lambda_{1} \sum_{m=1}^{N} \frac{1}{m !}\left\langle: \cdot{ }^{\otimes m}:, g_{1}^{\otimes m}\right\rangle \\
& +\lambda_{2} \sum_{m=1}^{N} \frac{1}{m !}\left\langle: \cdot{ }^{\otimes m}:, g_{2}^{\otimes m}\right\rangle \\
& \vdots \\
& +\lambda_{k} \sum_{m=1}^{N} \frac{1}{m !}\left\langle: \cdot{ }^{\otimes m}:, g_{k}^{\otimes m}\right\rangle,
\end{aligned}
$$

then, as $N \rightarrow \infty, F_{N} \rightarrow F$ in $\left(L^{2}\right)$ where $F=\lambda_{1}: e^{\left\langle\cdot, g_{1}\right\rangle}:+\lambda_{2}: e^{\left\langle\cdot, g_{2}\right\rangle}:+\cdots+\lambda_{k}: e^{\left\langle\cdot, g_{k}\right\rangle}:$. In our proof we will assume that $F_{N} \rightarrow F$ in $\mathcal{W}^{1 / 2}$. Also, $g$ will be taking on the form $g=$ $\sum_{j=1}^{k} \alpha_{j} 1_{\left[0, c_{j}\right)}, k \in \mathbb{N}, \alpha_{j}, c_{j} \in \mathbb{R}$. The formula will then be generalized to $\theta\left(X(t), F_{N}\right)$ with $F_{N}$ chosen as above. An extension to $\theta(X(t), F)$ with general $F \in \mathcal{W}^{1 / 2}$ will be achieved via a limiting process.

Proof of TheOrem 5.2. In the proof of [6, Theorem 1] which uses the $S$-transform, there are two components that were treated separately: (1) when $a \leq t \leq c$, and (2) when $c<t \leq b$.

Now suppose that $F=B(c), 0 \leq a \leq c \leq b$. We claim that our new formula in the above theorem is correct when we replace $F$ with $B(c)$. To see that this is correct, it is enough to show that $B(c) \in W^{1 / 2}$. We proceed by computing the norm for $B(c)$ in the space $\mathscr{W}^{1 / 2}$. Indeed, since $B(c)=\left\langle\cdot, 1_{[0, c)}\right\rangle$, we have

$$
\begin{aligned}
\partial_{s} B(c) & =1_{[0, c)}(s), \\
\|B(c)\|_{0}^{2} & =\left\|\left\langle\cdot, 1_{[0, c)}\right\rangle\right\|_{0}^{2}=\left|1_{[0, c)}\right|_{0}^{2} \\
& =\int_{\mathbb{R}} 1_{[0, c)}(s) d s=\int_{0}^{c} d s=c .
\end{aligned}
$$


Therefore,

$$
\|B(c)\|_{\mathbb{W}^{1 / 2}}=\|B(c)\|_{0}^{2}+\int_{a}^{b}\left\|\partial_{s} B(c)\right\|_{0}^{2} d s=c+(b-a) .
$$

Hence, $B(c) \in \mathcal{W}^{1 / 2}$. Now since $\partial_{s} B(c)=1_{[0, c)}(s)$, we then have

$$
\int_{a}^{t} f(s)\left(\partial_{s} B(c)\right) \frac{\partial^{2} \theta}{\partial x \partial y}(X(s), B(c)) d s= \begin{cases}\int_{a}^{t} f(s) \frac{\partial^{2} \theta}{\partial x \partial y}(X(s), B(c)) d s \quad \text { if } a \leq c, \\ \int_{a}^{c} f(s) \frac{\partial^{2} \theta}{\partial x \partial y}(X(s), B(c)) d s \quad \text { if } c<t \leq b .\end{cases}
$$

Thus, the two components (1) and (2) above in Theorem 3.1 are put together as one piece so as to satisfy the above theorem.

In general, suppose $a \leq c_{1} \leq c_{2} \leq \cdots \leq c_{p} \leq b$. Let $\tilde{\theta}\left(x, y_{1}, \ldots, y_{p}\right)$ be a function defined on $\mathbb{R}^{p+1}$ and of class $\mathscr{C}^{2}$. Then we have the following formula which is simply a generalization of the one above:

$$
\begin{aligned}
\tilde{\theta}\left(X(t), B\left(c_{1}\right), \ldots, B\left(c_{p}\right)\right)= & \tilde{\theta}\left(X(a), B\left(c_{1}\right), \ldots, B\left(c_{p}\right)\right) \\
& +\int_{a}^{t} \partial_{s}^{*}\left(f(s) \frac{\partial \tilde{\theta}}{\partial x}\left(X(s), B\left(c_{1}\right), \ldots, B\left(c_{p}\right)\right)\right) d s \\
& +\frac{1}{2} \int_{a}^{t} f(s)^{2} \frac{\partial^{2} \tilde{\theta}}{\partial x^{2}}\left(X(s), B\left(c_{1}\right), \ldots, B\left(c_{p}\right)\right) d s \\
& +\int_{a}^{t} f(s) \sum_{j=1}^{p}\left(\partial_{s} B\left(c_{j}\right)\right) \frac{\partial^{2} \tilde{\theta}}{\partial x \partial y_{j}}\left(X(s), B\left(c_{1}\right), \ldots, B\left(c_{p}\right)\right) d s .
\end{aligned}
$$

For a suitable function $G: \mathbb{R}^{p} \rightarrow \mathbb{R}$ and of class $\mathscr{C}^{2}$, we can transform the function $\tilde{\theta}$ to coincide with $\theta$ as a function defined on $\mathbb{R}^{2}$ in the following way:

$$
\tilde{\theta}\left(X(t), B\left(c_{1}\right), \ldots, B\left(c_{p}\right)\right)=\theta\left(X(t), G\left(B\left(c_{1}\right), \ldots, B\left(c_{p}\right)\right)\right)=\theta(x, y) .
$$

With this transformation and using the chain rule, the above integral equation then takes on the following form:

$$
\begin{aligned}
& \theta\left(X(t), G\left(B\left(c_{1}\right), \ldots, B\left(c_{p}\right)\right)\right) \\
&= \theta\left(X(a), G\left(B\left(c_{1}\right), \ldots, B\left(c_{p}\right)\right)\right) \\
&+ \int_{a}^{t} \partial_{s}^{*}\left(f(s) \frac{\partial \theta}{\partial x}\left(X(s), G\left(B\left(c_{1}\right), \ldots, B\left(c_{p}\right)\right)\right) d s\right. \\
&+ \frac{1}{2} \int_{a}^{t} f(s)^{2} \frac{\partial^{2} \theta}{\partial x^{2}}\left(X(s), G\left(B\left(c_{1}\right), \ldots, B\left(c_{p}\right)\right)\right) d s \\
&+ \int_{a}^{t} f(s) \sum_{j=1}^{p}\left(\partial_{s} B\left(c_{j}\right)\right) \frac{\partial^{2} \theta}{\partial x \partial y}\left(X(s), G\left(B\left(c_{1}\right), \ldots, B\left(c_{p}\right)\right)\right) \\
& \times \frac{\partial}{\partial y_{j}} G\left(B\left(c_{1}\right), \ldots, B\left(c_{p}\right)\right) d s .
\end{aligned}
$$


Now let

$$
\begin{aligned}
F_{N}= & \lambda_{1} \sum_{m=1}^{N} \frac{1}{m !}\left\langle:{ }^{\otimes m}:\left(\sum_{j=1}^{p^{(1)}} \alpha_{j}^{(1)} 1_{\left[0, c_{j}^{(1)}\right)}\right)^{\otimes m}\right\rangle \\
& +\lambda_{2} \sum_{m=1}^{N} \frac{1}{m !}\left\langle: \cdot{ }^{\otimes m}:\left(\sum_{j=1}^{p^{(2)}} \alpha_{j}^{(2)} 1_{\left[0, c_{j}^{(2)}\right)}\right)^{\otimes m}\right\rangle \\
& \vdots \\
& +\lambda_{k-1} \sum_{m=1}^{N} \frac{1}{m !}\left\langle: \cdot{ }^{\otimes m}:\left(\sum_{j=1}^{p^{(k-1)}} \alpha_{j}^{(k-1)} 1_{\left[0, c_{j}^{(k-1)}\right)}\right)^{\otimes m}\right\rangle \\
& +\lambda_{k} \sum_{m=1}^{N} \frac{1}{m !}\left\langle: \cdot \otimes m:\left(\sum_{j=1}^{p^{(k)}} \alpha_{j}^{(k)} 1_{\left[0, c_{j}^{(k)}\right)}\right)^{\otimes m}\right\rangle .
\end{aligned}
$$

We know that for any $n \in \mathbb{N}$,

$$
\left\langle: \otimes^{\otimes n}:, 1_{[0, c)}^{\otimes n}\right\rangle=\sum_{k=0}^{[n / 2]}\left(\begin{array}{c}
n \\
2 k
\end{array}\right)(2 k-1) ! !(-c)^{k} B(c)^{n-2 k}=\sum_{i=1}^{m} a_{i} B(c)^{i}
$$

for some constants $a_{i}$ and $m$. Some summands could as well be zero. This then becomes a polynomial in $B(c)$. We can similarly write $F_{N}$ as a polynomial in Brownian motion as follows:

$$
\begin{aligned}
F_{N}= & \lambda_{1} \sum_{m_{1}^{(1)} \cdots m_{p}^{(1)}} a_{m_{1}^{(1)} \cdots m_{p}^{(1)}} B\left(c_{1}^{(1)}\right)^{m_{1}^{(1)}} \cdots B\left(c_{p}^{(1)}\right)^{m_{p}^{(1)}} \\
& +\lambda_{2} \sum_{m_{1}^{(2)} \cdots m_{p}^{(2)}} a_{m_{1}^{(2)} \cdots m_{p}^{(2)}} B\left(c_{1}^{(2)}\right)^{m_{1}^{(2)}} \cdots B\left(c_{p}^{(2)}\right)^{m_{p}^{(2)}} \\
& \vdots \\
& +\lambda_{k} \sum_{m_{1}^{(k)} \cdots m_{p}^{(k)}} a_{m_{1}^{(k)} \cdots m_{p}^{(k)}} B\left(c_{1}^{(k)}\right)^{m_{1}^{(k)} \cdots B\left(c_{p}^{(k)}\right)^{m_{p}^{(k)}},}
\end{aligned}
$$

where again some coefficients $a_{m_{1}^{(j)} \ldots m_{p}^{(j)}}$ could be zero for some $j$. Suppose $F_{N}$ is restricted to only one summand out of the $k$ summands above, that is, suppose that

$$
F_{N}=\lambda_{1} \sum_{m_{1}^{(1)} \cdots m_{p}^{(1)}} a_{m_{1}^{(1)} \cdots m_{p}^{(1)}} B\left(c_{1}^{(1)}\right)^{m_{1}^{(1)}} \cdots B\left(c_{p}^{(1)}\right)^{m_{p}^{(1)}} .
$$

Then we see that the function $F_{N}$ is a good choice for our composition. That is, we let $F_{N}=G\left(B\left(c_{1}^{(1)}\right), \ldots, B\left(c_{p}^{(1)}\right)\right)$ so that the following ensues:

$$
\begin{aligned}
& \theta\left(X(t), G\left(B\left(c_{1}^{(1)}\right), \ldots, B\left(c_{p}^{(1)}\right)\right)\right) \\
& \quad=\theta\left(X(t), \lambda_{1} \sum_{m_{1}^{(1)} \cdots m_{p}^{(1)}} a_{m_{1}^{(1)} \cdots m_{p}^{(1)}} B\left(c_{1}^{(1)}\right)^{m_{1}^{(1)}} \cdots B\left(c_{p}^{(1)}\right)^{m_{p}^{(1)}}\right) .
\end{aligned}
$$


Moreover,

$$
\begin{aligned}
\frac{\partial}{\partial y_{j}} & G\left(B\left(c_{1}^{(1)}\right), \ldots, B\left(c_{p}^{(1)}\right)\right) \\
& =\lambda_{1} \sum_{m_{1}^{(1)} \cdots m_{p}^{(1)}} a_{m_{1}^{(1)} \cdots m_{p}^{(1)}} B\left(c_{1}^{(1)}\right)^{m_{1}^{(1)}} \cdots m_{j}^{(1)} B\left(c_{j}^{(1)}\right)^{m_{j}^{(1)}-1} \cdots B\left(c_{p}^{(1)}\right)^{m_{p}^{(1)}} .
\end{aligned}
$$

Therefore,

$$
\begin{aligned}
\theta\left(X(t), F_{N}\right)= & \theta\left(X(a), F_{N}\right)+\int_{a}^{t} \partial_{s}^{*}\left(f(s) \frac{\partial \theta}{\partial x}\left(X(s), F_{N}\right)\right) d s \\
& +\frac{1}{2} \int_{a}^{t} f(s)^{2} \frac{\partial^{2} \theta}{\partial x^{2}}\left(X(s), F_{N}\right) d s \\
& +\int_{a}^{t} \sum_{j=1}^{p} f(s)\left(\partial_{s} B\left(c_{j}^{(1)}\right)\right) \frac{\partial^{2} \theta}{\partial x \partial y}\left(X(s), F_{N}\right) \lambda_{1} \\
& \times \sum_{m_{1}^{(1)} \cdots m_{p}^{(1)}} a_{m_{1}^{(1)} \cdots m_{p}^{(1)}} B\left(c_{1}^{(1)}\right)^{m_{1}^{(1)}} \cdots m_{j}^{(1)} \\
& \times B\left(c_{j}^{(1)}\right)^{m_{j}^{(1)}-1} \cdots B\left(c_{p}^{(1)}\right)^{m_{p}^{(1)}} d s .
\end{aligned}
$$

Now, by the product rule,

$$
\partial_{s}(\varphi \psi)=\left(\partial_{s} \varphi\right) \psi+\varphi\left(\partial_{s} \psi\right)
$$

We then incorporate this rule into the last summand of the most recent equation (5.23) above to obtain

$$
\begin{aligned}
\sum_{j=1}^{p} f(s) & \frac{\partial^{2} \theta}{\partial x \partial y}\left(X(s), F_{N}\right) \lambda_{1} \sum_{m_{1}^{(1)} \cdots m_{p}^{(1)}} a_{m_{1}^{(1)} \cdots m_{p}^{(1)}} B\left(c_{1}^{(1)}\right)^{m_{1}^{(1)}} \cdots m_{j}^{(1)} \\
& \times B\left(c_{j}^{(1)}\right)^{m_{j}^{(1)}-1} \cdots B\left(c_{p}^{(1)}\right)^{(1)} \partial_{s} B\left(c_{j}^{(1)}\right) \\
= & f(s) \frac{\partial^{2} \theta}{\partial x \partial y}\left(X(s), F_{N}\right)\left(\partial_{s} F_{N}\right) .
\end{aligned}
$$

Then, by linearity, the above result can be extended to all the $k$ summands in the original expression for $F_{N}$. Hence for $F_{N} \in W^{1 / 2}$, the formula in our theorem is true; that is,

$$
\begin{aligned}
\theta\left(X(t), F_{N}\right)= & \theta\left(X(a), F_{N}\right)+\int_{a}^{t} \partial_{s}^{*}\left(f(s) \frac{\partial \theta}{\partial x}\left(X(s), F_{N}\right)\right) d s \\
& +\frac{1}{2} \int_{a}^{t} f(s)^{2} \frac{\partial^{2} \theta}{\partial x^{2}}\left(X(s), F_{N}\right) d s+\int_{a}^{t} f(s)\left(\partial_{s} F_{N}\right) \frac{\partial^{2} \theta}{\partial x \partial y}\left(X(s), F_{N}\right) d s .
\end{aligned}
$$

As stated earlier, by assumption, $F_{N} \rightarrow F$ as $N \rightarrow \infty$ in the $\mathcal{W}^{1 / 2}$-norm, where

$$
F=\lambda_{1}: e^{\left\langle\cdot, g_{1}\right\rangle}:+\lambda_{2}: e^{\left\langle\cdot, g_{2}\right\rangle}:+\cdots+\lambda_{k}: e^{\left\langle\cdot, g_{k}\right\rangle}:
$$


with

$$
g_{n}=\left(\sum_{j=1}^{p^{(n)}} \alpha_{j}^{(n)} 1_{\left[0, c_{j}^{(n)}\right)}\right)^{\otimes m}, \quad 1 \leq n \leq k .
$$

Therefore, there exists a subsequence $\left\{F_{N_{k}}\right\}_{k \geq 1} \subset\left\{F_{N}\right\}_{N \geq 1}$ such that $F_{N_{k}} \rightarrow F$ as $k \rightarrow \infty$ almost surely. For such a subsequence, the following is true:

$$
\begin{aligned}
\theta\left(X(t), F_{N_{k}}\right)= & \theta\left(X(a), F_{N_{k}}\right)+\int_{a}^{t} \partial_{s}^{*}\left(f(s) \frac{\partial \theta}{\partial x}\left(X(s), F_{N_{k}}\right)\right) d s \\
& +\frac{1}{2} \int_{a}^{t} f(s)^{2} \frac{\partial^{2} \theta}{\partial x^{2}}\left(X(s), F_{N_{k}}\right) d s+\int_{a}^{t} f(s)\left(\partial_{s} F_{N_{k}}\right) \frac{\partial^{2} \theta}{\partial x \partial y}\left(X(s), F_{N_{k}}\right) d s
\end{aligned}
$$

We claim that the following convergences hold almost surely as $k \rightarrow \infty$ :

$$
\begin{aligned}
\theta\left(X(t), F_{N_{k}}\right) & \rightarrow \theta(X(t), F), \\
\theta\left(X(a), F_{N_{k}}\right) & \rightarrow \theta(X(a), F), \\
\frac{1}{2} \int_{a}^{t} f(s)^{2} \frac{\partial^{2} \theta}{\partial x^{2}}\left(X(s), F_{N_{k}}\right) d s & \rightarrow \frac{1}{2} \int_{a}^{t} f(s)^{2} \frac{\partial^{2} \theta}{\partial x^{2}}(X(s), F) d s, \\
\int_{a}^{t} f(s)\left(\partial_{s} F_{N_{k}}\right) \frac{\partial^{2} \theta}{\partial x \partial y}\left(X(s), F_{N_{k}}\right) d s & \longrightarrow \int_{a}^{t} f(s)\left(\partial_{s} F\right) \frac{\partial^{2} \theta}{\partial x \partial y}(X(s), F) d s, \\
\int_{a}^{t} \partial_{s}^{*}\left(f(s) \frac{\partial \theta}{\partial x}\left(X(s), F_{N_{k}}\right)\right) d s & \longrightarrow \int_{a}^{t} \partial_{s}^{*}\left(f(s) \frac{\partial \theta}{\partial x}(X(s), F)\right) d s .
\end{aligned}
$$

Proof OF (5.30) and (5.31). Because of continuity of $\theta$, since $F_{N_{k}} \rightarrow F$ almost surely, those two convergences also hold almost surely.

Proof of (5.32). Let $\omega \in \mathscr{S}^{\prime}(\mathbb{R})$ be fixed. Since $F_{N_{k}}$ converges, it is a bounded sequence, that is, there exists $M>0$ such that $\left|F_{N_{k}}(\omega)\right| \leq M$ for all $k$. Therefore, the function given by $\partial^{2} \theta / \partial x^{2}:[a, b] \times[-M, M] \rightarrow \mathbb{R}$ is continuous on compact sets and hence uniformly continuous. Thus given $\epsilon>0$, there exists $\delta>0$ such that whenever $\left|x_{1}-x_{2}\right|^{2}+\left|y_{1}-y_{2}\right|^{2}<\delta$, we have

$$
\left|\frac{\partial^{2} \theta}{\partial x^{2}}\left(x_{1}, y_{1}\right)-\frac{\partial^{2} \theta}{\partial x^{2}}\left(x_{2}, y_{2}\right)\right|<\frac{2 \epsilon}{\|f\|_{\infty}^{2}(t-a)}
$$

Also, by the convergence of $\left\{F_{N_{k}}\right\}_{k \geq 1}$, there exists a number $N(\epsilon)$ depending on $\epsilon$ such that

$$
k \geq N(\epsilon) \Longrightarrow\left|F_{N_{k}}(\omega)-F(\omega)\right|<\delta,
$$


which implies that

$$
\begin{aligned}
& \frac{1}{2}\left|\int_{a}^{t} f(s)^{2}\left(\frac{\partial^{2} \theta}{\partial x^{2}}\left(X(s), F_{N_{k}}(\omega)\right)-\frac{\partial^{2} \theta}{\partial x^{2}}(X(s), F(\omega))\right) d s\right| \\
& \quad \leq \frac{\|f\|_{\infty}^{2}}{2} \int_{a}^{t}\left|\frac{\partial^{2} \theta}{\partial x^{2}}\left(X(s), F_{N_{k}}(\omega)\right)-\frac{\partial^{2} \theta}{\partial x^{2}}(X(s), F(\omega))\right| d s \\
& \quad<\frac{\|f\|_{\infty}^{2}}{2} \frac{2 \epsilon}{\|f\|_{\infty}^{2}(t-a)}(t-a) \\
& \quad=\epsilon
\end{aligned}
$$

and so (5.32) is proved.

Proof OF (5.33). It is known that in a Hilbert space $H$, if $x_{n} \rightarrow x$ and $y_{n} \rightarrow y$, then $\left\langle x_{n}, y_{n}\right\rangle \rightarrow\langle x, y\rangle$ where $\langle\cdot, \cdot\rangle$ is the inner product on $H$. By taking $H=L^{2}([a, t])$ with the Lebesgue measure it follows then that

$$
\begin{aligned}
\int_{a}^{t} f(s)\left(\partial_{s} F_{N_{k}}(\omega)\right) \frac{\partial^{2} \theta}{\partial x \partial y}\left(X(s), F_{N_{k}}(\omega)\right) d s & \equiv\left\langle\partial \cdot F_{N_{k}}(\omega), f(\cdot) \frac{\partial^{2} \theta}{\partial x \partial y}\left(X(\cdot), F_{N_{k}}(\omega)\right)\right\rangle, \\
\int_{a}^{t} f(s)\left(\partial_{s} F(\omega)\right) \frac{\partial^{2} \theta}{\partial x \partial y}(X(s), F(\omega)) d s & \equiv\left\langle\partial \cdot F(\omega), f(\cdot) \frac{\partial^{2} \theta}{\partial x \partial y}(X(\cdot), F(\omega))\right\rangle .
\end{aligned}
$$

By the same uniform continuity argument used above in proving (5.33), as $k \rightarrow \infty$, we see that

$$
f(\cdot) \frac{\partial^{2} \theta}{\partial x \partial y}\left(X(\cdot), F_{N_{k}}(\omega)\right) \rightarrow f(\cdot) \frac{\partial^{2} \theta}{\partial x \partial y}(X(\cdot), F(\omega))
$$

in $L^{2}([a, t])$.

Now $F_{N_{k}} \rightarrow F$ in $\mathscr{W}^{1 / 2}$. Hence $F_{N_{k}} \rightarrow F$ in $\left(L^{2}\right)$ and $\int_{a}^{t}\left\|\partial_{s} F_{N_{k}}-\partial_{s} F\right\|_{0}^{2} d s \rightarrow 0$ as $k \rightarrow \infty$. Therefore a change of integrals is justified and the following is true:

$$
\begin{aligned}
\int_{\mathscr{I}^{\prime}(\mathbb{R})} & \int_{a}^{t}\left|\partial_{s} F_{N}(\omega)-\partial_{s} F(\omega)\right|^{2} d s d \mu(\omega) \\
& =\int_{a}^{t} \int_{\mathscr{S}^{\prime}(\mathbb{R})}\left|\partial_{s} F_{N}(\omega)-\partial_{S} F(\omega)\right|^{2} d \mu(\omega) d s \\
& =\int_{a}^{t}\left\|\partial_{s} F_{N}-\partial_{s} F\right\|_{\left(L^{2}\right)}^{2} d s \rightarrow 0 .
\end{aligned}
$$

Let

$$
\int_{a}^{t}\left|\partial_{s} F_{N_{k}}(\omega)-\partial_{s} F(\omega)\right|^{2} d s=H_{N_{k}}(\omega)
$$

Then, $\left\{H_{N_{k}}(\omega)\right\}_{k \geq 1}$ is a sequence of positive functions and by the above result, $H_{N_{k}}(\omega) \rightarrow 0$ in $L^{1}(\mu)$. Therefore,

$$
\partial . F_{N_{k}}(\omega) \longrightarrow \partial . F(\omega)
$$

in $L^{2}([a, t])$ and so (5.33) is true. 
Proof OF (5.34). We approximate the $\left(L^{2}\right)$-norm of the difference of the two integrals. We claim the norm of this difference goes to zero. For convenience, use the following notation. Let

$$
\begin{aligned}
f(s) \frac{\partial \theta}{\partial x}\left(X(s), F_{N_{k}}(\omega)\right) & =\varphi_{N_{k}}(s), \\
f(s) \frac{\partial \theta}{\partial x}(X(s), F(\omega)) & =\varphi(s) .
\end{aligned}
$$

Then by Theorem 4.5, we have

$$
\begin{aligned}
& \left\|\int_{a}^{t} \partial_{s}^{*} \varphi_{N_{k}} d s-\int_{a}^{t} \partial_{s}^{*} \varphi d s\right\|_{0}^{2} \\
& \quad=\int_{a}^{t}\left\|\varphi_{N_{k}}-\varphi\right\|_{0}^{2} d s+\int_{a}^{t} \int_{a}^{t}\left(\left(\partial_{s} \varphi_{N_{k}}(u)-\partial_{s} \varphi(u), \partial_{u} \varphi_{N_{k}}(s)-\partial_{u} \varphi(s)\right)\right)_{0} d u d s,
\end{aligned}
$$

where $((\cdot, \cdot))_{0}$ is the inner product on $\left(L^{2}\right)$.

Since $F_{N_{k}}(\omega) \rightarrow F(\omega)$ in $\left(L^{2}\right)$, we can choose a subsequence so that the convergence is almost surely. Thus for such a subsequence, there exists $\Omega_{0}$ such that $P\left\{\Omega_{0}\right\}=1$ and for $\omega$ fixed, we have $F_{N_{k}}(\omega) \rightarrow F(\omega)$. Then, by the mean value theorem, for each $s$,

$$
\begin{aligned}
& \left|\frac{\partial \theta}{\partial x}\left(X(s), F_{N_{k}}(\omega)\right)-\frac{\partial \theta}{\partial x}(X(s), F(\omega))\right| \\
& \quad=\left|\frac{\partial^{2} \theta}{\partial x \partial y}(X(s), \xi(\omega))\right| \cdot\left|F_{N_{k}}(\omega)-F(\omega)\right|,
\end{aligned}
$$

where $\xi(\omega)$ is between $F_{N_{k}}(\omega)$ and $F(\omega)$. Since $\theta \in \mathscr{C}_{b}^{2}\left(\mathbb{R}^{2}\right),\left|\left(\partial^{2} \theta / \partial x \partial y\right)(X(s), \xi(\omega))\right|$ $<C$ for some constant $C$. So,

$$
\left\|\frac{\partial \theta}{\partial x}\left(X(s), F_{N_{k}}(\omega)\right)-\frac{\partial \theta}{\partial x}(X(s), F(\omega))\right\|_{0} \leq C\left\|F_{N_{k}}(\omega)-F(\omega)\right\|_{0} .
$$

As earlier noted, the convergence of the subsequence $\left\{F_{N_{k}}\right\}_{k \geq 1}$ of numbers implies that for some fixed constant $M>0$, the quantity $\left\|F_{N_{k}}(\omega)-F(\omega)\right\|_{0} \leq M$, for all $k$. Therefore, using the fact that $F_{N_{k}}(\omega) \rightarrow F(\omega)$ in $\left(L^{2}\right)$, we have

$$
\left\|\varphi_{N_{k}}-\varphi\right\|_{0} \leq\|f\|_{\infty} C\left\|F_{N_{k}}-F\right\|_{0} \longrightarrow 0
$$

and also

$$
\left\|\varphi_{N_{k}}-\varphi\right\|_{0} \leq\|f\|_{\infty} C\left\|F_{N_{k}}-F\right\|_{0} \leq\|f\|_{\infty} C M
$$

Since this bound is independent of both $s$ and $k$, by the bounded convergence theorem, as $k \rightarrow \infty$,

$$
\int_{a}^{t}\left\|\varphi_{N_{k}}-\varphi\right\|_{0}^{2} d s \rightarrow 0
$$


The convergence of the second summand of the right-hand side of (5.44) goes as follows:

$$
\begin{aligned}
& \left|\int_{a}^{t} \int_{a}^{t}\left(\left(\partial_{s} \varphi_{N_{k}}(u)-\partial_{s} \varphi(u), \partial_{u} \varphi_{N_{k}}(s)-\partial_{u} \varphi(s)\right)\right)_{0} d u d s\right| \\
& \quad \leq \int_{a}^{t} \int_{a}^{t}\left\|\partial_{s} \varphi_{N_{k}}(u)-\partial_{s} \varphi(u)\right\|_{0}\left\|\partial_{u} \varphi_{N_{k}}(s)-\partial_{u} \varphi(s)\right\|_{0} d u d s \\
& \quad \leq \frac{1}{2} \int_{a}^{t} \int_{a}^{t}\left(\left\|\partial_{s} \varphi_{N_{k}}(u)-\partial_{s} \varphi(u)\right\|_{0}^{2}+\left\|\partial_{u} \varphi_{N_{k}}(s)-\partial_{u} \varphi(s)\right\|_{0}^{2}\right) d u d s \\
& \quad=\int_{a}^{t} \int_{a}^{t}\left\|\partial_{u} \varphi_{N_{k}}(s)-\partial_{u} \varphi(s)\right\|_{0}^{2} d u d s \\
& \quad \leq \int_{a}^{t}\left(\int_{\mathbb{R}}\left\|\partial_{u} \varphi_{N_{k}}(s)-\partial_{u} \varphi(s)\right\|_{0}^{2} d u\right) d s .
\end{aligned}
$$

The number operator, $N$ can also be expressed as $N=\int_{\mathbb{R}} \partial_{s}^{*} \partial_{s} d s$ (see [6]). Hence, for any $\varphi \in L^{2}\left([a, b] ; W^{1 / 2}\right)$, we have

$$
\begin{aligned}
\left\|N^{1 / 2} \varphi\right\|_{0}^{2} & =((N \varphi, \varphi))_{0}=\int_{\mathbb{R}}\left(\left(\partial_{s}^{*} \partial_{s} \varphi, \varphi\right)\right)_{0} d s \\
& =\int_{\mathbb{R}}\left(\left(\partial_{s} \varphi, \partial_{s} \varphi\right)\right)_{0} d s=\int_{\mathbb{R}}\left\|\partial_{s} \varphi\right\|_{0}^{2} d s .
\end{aligned}
$$

Therefore, by this result,

$$
\int_{a}^{t}\left(\int_{\mathbb{R}}\left\|\partial_{u} \varphi_{N_{k}}(s)-\partial_{u} \varphi(s)\right\|_{0}^{2} d u\right) d s=\int_{a}^{t}\left\|N^{1 / 2} \partial_{u} \varphi_{N_{k}}(s)-\partial_{u} \varphi(s)\right\|_{0}^{2} d s .
$$

But $N^{1 / 2}$ is a bounded operator from $\mathcal{W}^{1 / 2}$ into $\left(L^{2}\right)$. Therefore, by the same mean value theorem argument, since $F_{N_{k}} \rightarrow F$ also in $W^{1 / 2}$ by our original assumption, we have

$$
\left|\int_{a}^{t} \int_{a}^{t}\left(\left(\partial_{s} \varphi_{N_{k}}(u)-\partial_{s} \varphi(u), \partial_{u} \varphi_{N_{k}}(s)-\partial_{u} \varphi(s)\right)\right)_{0} d u d s\right| \rightarrow 0
$$

as $k \rightarrow \infty$.

Therefore, it has been shown that the convergence in (5.34) is true in the $\left(L^{2}\right)$ norm. We then pick a further subsequence of the subsequence $\left\{F_{N_{k}}\right\}_{k \geq 1}$ also denoted by $\left\{F_{N_{k}}\right\}_{k \geq 1}$ such that convergence hold almost surely. This then proves all the convergences necessary and completes the proof of the theorem.

\section{REFERENCES}

[1] W. G. Cochran, H.-H. Kuo, and A. Sengupta, A new class of white noise generalized functions, Infin. Dimens. Anal. Quantum Probab. Relat. Top. 1 (1998), no. 1, 43-67.

[2] M. Hitsuda, Formula for Brownian partial derivatives, Proceedings of the 2nd Japan-USSR Symposium on Probability Theory, vol. 2, Kyoto University, Kyoto, 1972, pp. 111114.

[3] K. Itô, Multiple Wiener integral, J. Math. Soc. Japan 3 (1951), 157-169.

[4] Extension of stochastic integrals, Proceedings of the International Symposium on Stochastic Differential Equations (Res. Inst. Math. Sci., Kyoto Univ., Kyoto, 1976), Wiley, New York, 1978, pp. 95-109. 
[5] I. Kubo and S. Takenaka, Calculus on Gaussian white noise. III, Proc. Japan Acad. Ser. A Math. Sci. 57 (1981), no. 9, 433-437.

[6] H.-H. Kuo, White Noise Distribution Theory, Probability and Stochastics Series, CRC Press, Florida, 1996.

[7] D. Nualart, The Malliavin Calculus and Related Topics, Probability and Its Applications, Springer-Verlag, New York, 1995.

[8] N. Obata, White Noise Calculus and Fock Space, Lecture Notes in Mathematics, vol. 1577, Springer-Verlag, Berlin, 1994.

[9] N. Prevault, An Extension of the Hitsuda-Itô Formula, Equipe d'Analyse et Probabilités, Université d'Evry-Val d'Essonne, France, 1997.

SAID Ngobi: Alabama STATE UnIVERSITy, Box 161, Montgomery, AL 36101, USA

E-mail address: ngobi@math1ab.a1asu.edu 


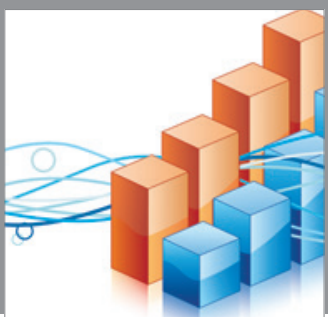

Advances in

Operations Research

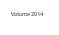

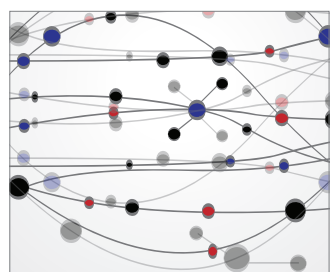

\section{The Scientific} World Journal
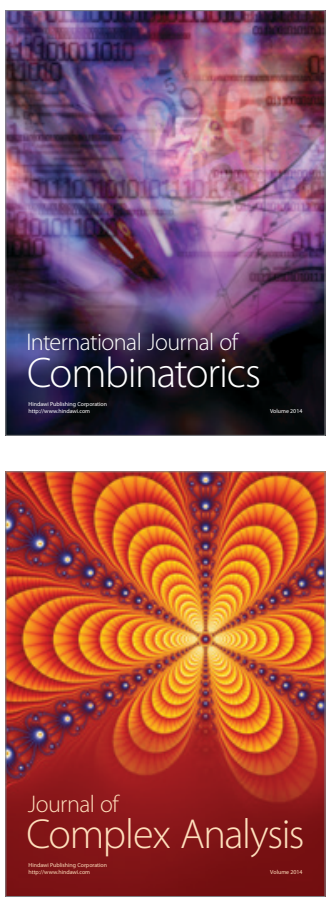

International Journal of

Mathematics and

Mathematical

Sciences
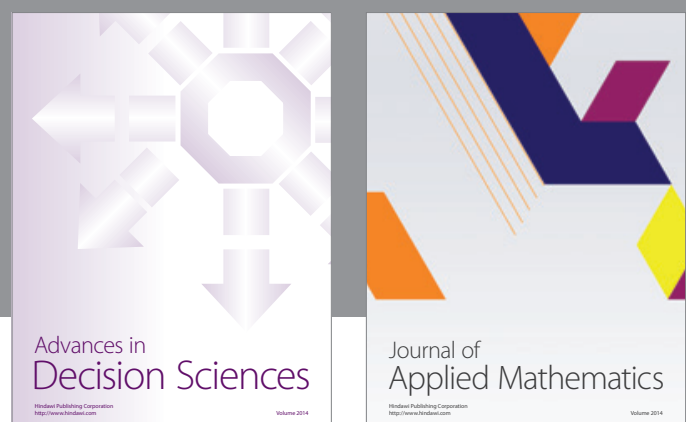

Journal of

Applied Mathematics
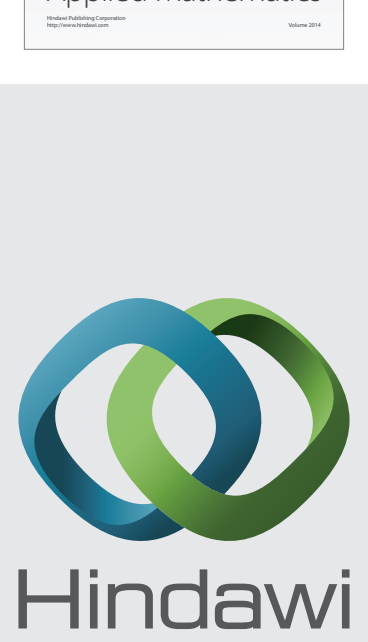

Submit your manuscripts at http://www.hindawi.com
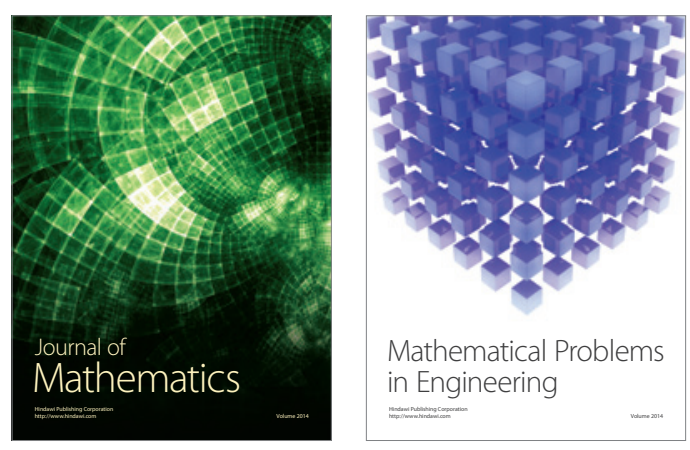

Mathematical Problems in Engineering
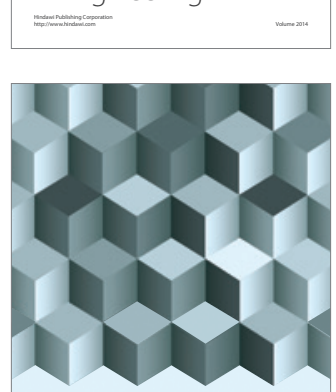

Journal of

Function Spaces
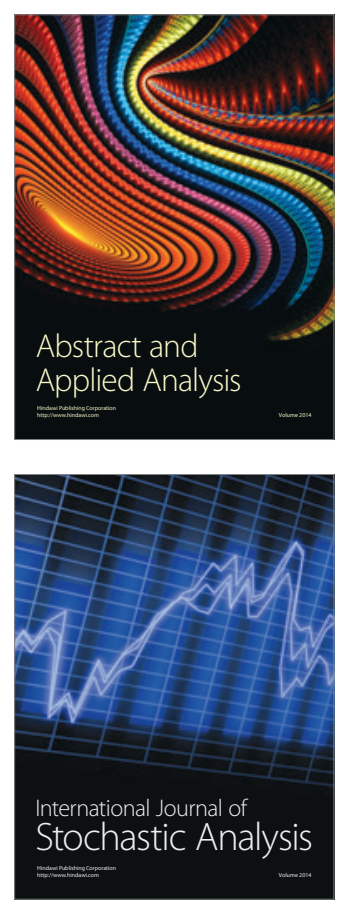

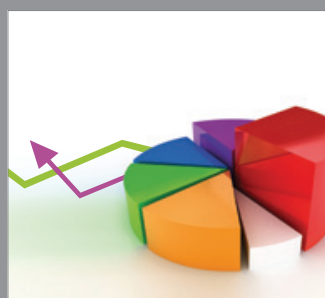

ournal of

Probability and Statistics

Promensencen
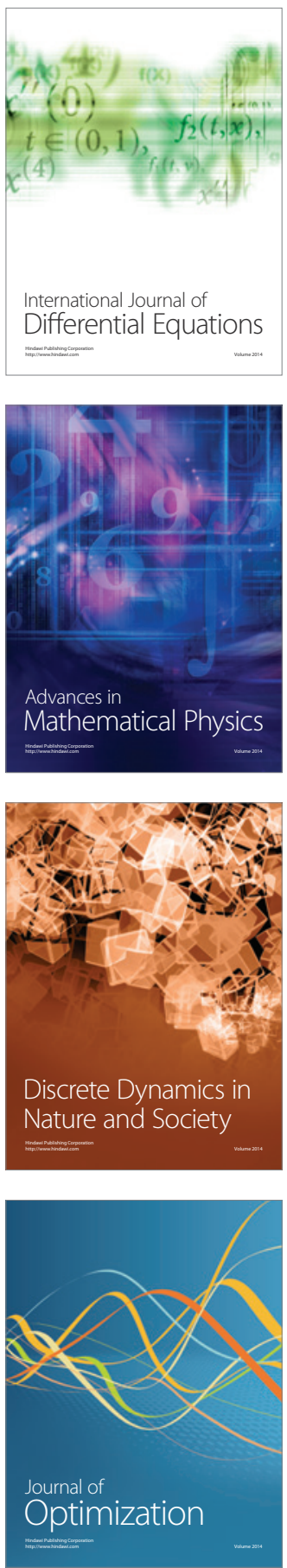